\title{
Article \\ Corn Grain and Stover Nutrient Uptake Responses from Sandy Soil Treated with Designer Biochars and Compost
}

\author{
Jeffrey M. Novak*(D), Donald W. Watts $\mathbb{D}$, Gilbert C. Sigua and Thomas F. Ducey \\ United States Department of Agriculture (USDA), Agricultural Research Service (ARS), Coastal Plain Soil, \\ Water and Plant Research Center, 2611 West Lucas Street, Florence, SC 29501, USA; \\ don.watts@usda.gov (D.W.W.); gilbert.sigua@usda.gov (G.C.S.); tom.ducey@usda.gov (T.F.D.) \\ * Correspondence: Jeff.novak@usda.gov
}

check for updates

Citation: Novak, J.M.; Watts, D.W.; Sigua, G.C.; Ducey, T.F. Corn Grain and Stover Nutrient Uptake Responses from Sandy Soil Treated with Designer Biochars and Compost. Agronomy 2021, 11, 942. https:// doi.org/10.3390/agronomy11050942

Academic Editor: Domenico Ronga

Received: 9 March 2021

Accepted: 4 May 2021

Published: 10 May 2021

Publisher's Note: MDPI stays neutral with regard to jurisdictional claims in published maps and institutional affiliations.

Copyright: (c) 2021 by the authors. Licensee MDPI, Basel, Switzerland. This article is an open access article distributed under the terms and conditions of the Creative Commons Attribution (CC BY) license (https:// creativecommons.org/licenses/by/ $4.0 /)$.

\begin{abstract}
Biochars are used for soil fertility improvement because they may contain certain elements that plants use as nutrients. However, few studies have demonstrated enhanced crop nutrient uptake. Our study examined nutrient uptake responses of corn (Zea Mays L.) grain and stover over 4 years (Y) after a Goldsboro sandy loam (fine-loamy, siliceous, sub-active, thermic Aquic Paleudults) received different designer biochars and a compost. The designer biochars were produced from lodgepole pine (Pinus contorta) chip (PC), poultry litter (PL), blends with switchgrass (SG; Panicum virgatum), and a SG compost alone. Topsoil treated with 100\% PL biochar and blended PC:PL biochar had significantly greater Mehlich 1 (M1) extractable P, K and Na contents compared to the control or other treatments. No significant differences were detected in annual grain nutrient concentrations. In the first corn stover harvest $\left(\mathrm{Y}_{1}\right)$, significantly greater concentrations of $\mathrm{P}$ and $\mathrm{K}$ were taken up after treatment with 100\% PL biochar, with PC:PL blend and with SG when compared to control. By the fourth corn stover harvest $\left(\mathrm{Y}_{4}\right)$, nutrient uptake between treatments was not significantly different. Biochar impact on corn stover $\mathrm{P}, \mathrm{K}$ and Na concentrations was time dependent, suggesting that repeated biochar applications may be needed.
\end{abstract}

Keywords: corn grain; corn stover; designer biochar; nutrient uptake

\section{Introduction}

Biochar has global attention as a soil amendment because it can replenish nutrient concentrations in poor-fertility soils [1-3]. Biochars supply inorganic nutrients to soils because they contain $\mathrm{N}$ and $\mathrm{S}$ associated with organic materials [4] and by having micro (e.g., $\mathrm{Cu}$ and $\mathrm{Zn}$ ) and macro-elements (e.g., P and K) associated with the ash content [5-7]. These macro- and micro-elements added to soil are then available for plant nutrient uptake [7]. As reviewed by Hossain et al. [8], biochars can also influence the mobility $[9,10]$ and bioavailability [11] of nutrients, as well as impact nutrient cycling [12]. Thus, the ability of biochars to act as a satisfactory soil fertility amendment is highly dependent on crops being able to uptake those nutrients for better plant growth and yield improvements [13].

Many biochar-plant response studies have been conducted at the laboratory, greenhouse and field scales [1,14]. In these studies, biochar impact on crop growth and yield characteristics was variable and complex [6,7]. Variable crop responses to biochar additions have been linked to inconsistent nutrient quantities contained within initial feedstocks [4,15], pyrolysis conditions [5], biochar morphology [16], and differences in soil types [6]. For example, biochars produced from lignocellulosic-based feedstocks usually have lower concentrations of total $\mathrm{P}$ and $\mathrm{K}$ as compared to animal manure-based biochars $[4,5]$. A higher pyrolysis temperature used in feedstock carbonization processes amasses element concentrations in the ash fraction [4,5]. With respect to biochar morphology, Novak et al. [16] showed that more dissolved $\mathrm{P}$ was released in water leachate from PL biochar as dust sized $(<0.42 \mathrm{~mm})$ compared to pellets $(>2 \mathrm{~mm})$. The ability of biochars to improve soil quality has also been linked to soil textural differences $[14,17]$ 
and variations in climatic conditions (i.e., tropics vs. temperate regions; [18]. Thus, the inconsistent nutrient concentrations and application of biochars to different soils types under variable climatic conditions exacerbate the confusion in selecting a biochar type (i.e., feedstock source, pyrolysis temperature) for fertility management $[2,19]$ and crop yield improvement [13].

To better optimize biochar selection as a soil amendment, Novak et al. [16,20] developed the designer biochar concept that states the biochar should have specific properties that target a particular soil deficiency. Consequently, designer biochars can be produced with specific chemical (e.g., $\mathrm{pH}$ and high $\mathrm{P}$ and $\mathrm{K}$ contents,) and physical properties (e.g., pellets and flakes) through choosing a particular feedstock, pyrolysis conditions, and biochar morphology [21]. As such, the specific soil deficiency is first measured or identified, then a biochar is designed with characteristics that addresses the targeted soil deficiency. Furthermore, if the biochar has properties that are not advantageous (e.g., alkaline $\mathrm{pH}$, high nutrient contents, and excessive salts) for improving soil-plant relationships, then the biochar can be blended with compost or other biochar types to compensate for these properties of concern. Reports have shown that compost added to biochars enhances nutrient retention [22] and stimulates nutrient bioavailability [23].

Therefore, we utilized the designer biochar concept to produce biochar types that had specific properties to improve soil fertility in a Goldsboro loamy sand. These amendments were produced from 100\% PC, 100\% PL, 2:1 PC:PL blend, 2:1 PC:SG compost blend, and $100 \%$ SG compost and were chosen specifically to compensate for the low soil phosphorus $(\mathrm{P})$ and potassium $(\mathrm{K})$ contents in Goldsboro soils, blended to reduce excessive P contents in PL biochar, and co-blended with compost to enhance nutrient bioavailability. After biochar and compost application to field plots, multi-cycles of corn (Zea mays L.) were grown and these amendments impact on grain yields and biomass production was reported [24]. In that study, no improvement was described in corn grain yields or stover production. However, biochars and stover impacts on nutrient uptake dynamics were still in question. Inorganic fertilizer was added to all plots at typical fertilizer management rates to ensure that Goldsboro soils had sufficient nutrients if the biochar/compost mixtures did not supply critical plant nutrients. Here, we report the influence of these amendments on annual nutrient uptake by corn grain and stover along with topsoil Mehlich 1 extractable nutrient concentrations. Our hypothesis was that corn grain and stover nutrient uptake should increase in response to soil nutrient improvements after designer biochar application.

\section{Materials and Methods}

\subsection{Site Characteristics and Soil Properties}

The field plots for this experiment were located on grounds of the United States Department of Agriculture, Agricultural Research Service, Coastal Plain Soil, Water, and Plant Research Center, Florence, South Carolina, USA $\left(34^{\circ} 14^{\prime} 38^{\prime \prime} \mathrm{N}\right.$ and $\left.79^{\circ} 48^{\prime} 45.3^{\prime \prime} \mathrm{W}\right)$. For nearly six decades, this field was used for agricultural farming research involving buried trickle irrigation, colored mulch technology, and tillage research on vegetables and row crops production yields. Prior tillage operations in this field consisted of conservation tillage that entailed only deep tilling using a shank to rip the soil to $40 \mathrm{~cm}$ soil depth, while conventional tillage involved disking soil to approximately $10 \mathrm{~cm}$ depth. A Goldsboro soil series encompasses the experimental field. The topsoil ( $0-15 \mathrm{~cm}$ deep) of the Goldsboro soil is composed of $67.8 \%$ sand, $21.9 \%$ silt, and $10.3 \%$ clay by the hydrometer method [25]. According to the USDA textural classification, this soil is a sandy loam. This soil series is a moderately well-drained Ultisol and formed in marine deposits, interlaced with fluviomarine sediments [26].

\subsection{Designer Biochar Production and Chemical Characterization}

Designer biochar production technology and chemical characterization results have been described [24]. Briefly, the 100\% PC and 100\% PL biochar were commercially available. The SG crop was obtained from plots grown at the Clemson University Pee Dee Research 
and Education Center, Darlington, South Carolina under the management of Dr. Jim Frederick (Clemson University, Pee Dee Research and Education Center, Darlington, SC, USA). After harvest, the SG was pulverized into $6 \mathrm{~mm}$ flakes and allowed to compost for one week prior to blending with $100 \%$ PC biochar. Biochars and compost were characterized for their $\mathrm{pH}$, ash, and C, H, O, N contents [24]. The 100\% PC and 100\% PL biochars total elemental analysis was determined by ashing the material at $600^{\circ} \mathrm{C}$, digesting the ash using ASTM method SW866 [27] and quantifying $\mathrm{P}, \mathrm{K}, \mathrm{S}, \mathrm{Mn}, \mathrm{Cu}, \mathrm{Fe}, \mathrm{Na}, \mathrm{Mg}$, and $\mathrm{Ca}$ concentrations using ICP-OES (Table 1). For the compost, its $\mathrm{P}, \mathrm{K}, \mathrm{Cu}$, and $\mathrm{Zn}$ contents were determined by acid digestion by the Clemson University Agricultural Service Laboratory [28]. Chemical compositions for the biochar 2:1 blends were estimated based on field mixture ratios and using their measured chemical contents.

Table 1. Chemical composition of designer biochars and compost (PC = pine chips; PL = poultry litter; SG = switchgrass compost; units are $\mathrm{mg} \mathrm{kg}^{-1}$, dry-basis).

\begin{tabular}{cccccc}
\hline Parameter & $\mathbf{1 0 0} \% \mathbf{P C}$ & $\mathbf{1 0 0} \% \mathbf{P L}$ & PC:PL 2:1 & PC:SG 2:1 & SG \\
\hline $\mathrm{P}$ & 250 & 33,600 & 11,367 & 2973 & 700 \\
$\mathrm{~K}$ & 3010 & 72,300 & 26,107 & 4627 & 2900 \\
$\mathrm{~S}$ & 110 & 18,100 & 6118 & 272 & 400 \\
$\mathrm{Mn}$ & 262 & 1210 & 578 & 189 & 44 \\
$\mathrm{Cu}$ & 190 & 1600 & 660 & 129 & 7 \\
$\mathrm{Fe}$ & 820 & 4460 & 2033 & 569 & 67 \\
$\mathrm{Na}$ & 1240 & 20,400 & 7627 & 837 & 30 \\
$\mathrm{Mg}$ & 1490 & 16,700 & 6560 & 1360 & 1100 \\
$\mathrm{Ca}$ & 6190 & 46,700 & 19,693 & 4627 & 1500 \\
\hline
\end{tabular}

\subsection{Establishing and Managing Field Plots}

\subsubsection{Establishing Plots and Soil Sampling}

Twenty-four plots $\left(40 \mathrm{~m}^{2}\right)$ were established in a randomized complete block design in December 2015 as described [24]. In January 2016, the first-year $\left(Y_{1}\right)$ soil samples were randomly collected at $0-15 \mathrm{~cm}$ depths for fertility analyses from all plots and treatments. Mehlich 1 extractable soil nutrients in these soil samples were extracted and quantified using ICP-OES (Spectro-ICP, Ametek Analytical Instruments, Kleve, Germany) for P, K, Mn, $\mathrm{Cu}, \mathrm{Fe}, \mathrm{Na}, \mathrm{Mg}$, and $\mathrm{Ca}$ by the Clemson University Agricultural Service Laboratory [28]. The soil sampling and fertility measurements were repeated in a similar manner for year $2\left(Y_{2}-2017\right)$, year $3\left(Y_{3}-2018\right)$, year $4\left(Y_{4}-2019\right)$ and year $5\left(Y_{5}-2020-\right.$ Table 2$)$. While no corn was planted in $Y_{5}$, soil samples in $Y_{5}$ were collected and fertility was measured to determine soil characteristics at the end of this study.

Table 2. Research timeline for collection of soils for fertility measurements, corn planting and grain/stover harvesting dates (biochar applied to plots on 1-2 February 2016; na = not available).

\begin{tabular}{cccc}
\hline & & \multicolumn{2}{c}{ Corn Dates } \\
\hline $\begin{array}{c}\text { Soils Collection } \\
\text { Date }\end{array}$ & $\begin{array}{c}\text { Year (Y) of Study for } \\
\text { Soil }\end{array}$ & Planted & $\begin{array}{c}\text { Grain/Stover } \\
\text { Harvested }\end{array}$ \\
\hline 6-8 January 2016 & $\mathrm{Y}_{1}$ & 6 April 2016 & 9 September 2016 \\
12-18 January 2017 & $\mathrm{Y}_{2}$ & 5 April 2017 & 5-6 September 2017 \\
15 February 2018 & $\mathrm{Y}_{3}$ & 12 April 2018 & 30 August 2018 \\
15-18 March 2019 & $\mathrm{Y}_{4}$ & 17 April 2019 & 3 September 2019 \\
6-8 April 2020 & $\mathrm{Y}_{5}$ & na & na \\
\hline
\end{tabular}

\subsubsection{Biochar Application}

Biochar and compost were hand applied to plots in February 2016 and then lightly raked into the soil. Each plot received the equivalent of 30 tons ha $^{-1}$ of biochar or compost with the blends receiving 20 tons ha ${ }^{-1}$ of biochar mixed with 10 tons ha ${ }^{-1}$ of SG compost. 
Shortly thereafter, the amendments were mixed to a depth of $10 \mathrm{~cm}$ using a field disk cultivator. Control plots of Goldsboro sandy loam soil received no biochar application but were fertilizer with inorganic fertilizer (as described below).

\subsubsection{Agronomic Management}

Each plot in the period 2016-2018 received inorganic N-P-K fertilizer as needed in late March to early April at rates described [24]. For 2019, all plots received $132 \mathrm{~kg} \mathrm{~N} \mathrm{ha}^{-1}$, $31 \mathrm{~kg} \mathrm{P} \mathrm{ha}^{-1}$, and $95 \mathrm{~kg} \mathrm{ha}^{-1}$ of K. Corn (DKC64-89 variety) was planted in early to midApril (Table 1) at stand counts of 59,406 plants ha ${ }^{-1}$. Corn grain was harvested in late August to early September using a mechanical harvester on two center rows of each plot. After grain harvest, corn stover (leaves, cobs, and husks only) was collected by physically harvesting a row section in the two center corn rows. After oven drying $\left(3-5\right.$ days at $\left.65^{\circ} \mathrm{C}\right)$, corn stover was processed and yields reported on dry wt. basis in $\mathrm{kg} \mathrm{ha}^{-1}$. Monthly precipitation data for the period 2016-2020 were collected and organized (Table 3) from the database available at [29]. No supplemental irrigation was applied to the plots.

Table 3. Monthly and annual precipitation totals for study period.

\begin{tabular}{cccccc}
\hline \multicolumn{5}{c}{ Precipitation $(\mathbf{m m})$} \\
\hline Month & $\mathbf{2 0 1 6 +}$ & $\mathbf{2 0 1 7}+$ & $\mathbf{2 0 1 8}+$ & $\mathbf{2 0 1 9}$ & $\mathbf{2 0 2 0}$ \\
\hline January & 57.7 & 81.3 & 72.1 & 95.5 & 97.8 \\
February & 153.9 & 48 & 35.6 & 61.5 & 147.1 \\
March & 45.7 & 50.5 & 347 & 90.2 & 158 \\
April & 73.1 & 104.1 & 126.4 & 115.3 & 45.2 \\
May & 108.1 & 46.9 & 129.5 & 41.9 & 281.7 \\
June & 96.1 & 170.1 & 88.4 & 98 & 121.9 \\
July & 213.1 & 199.6 & 122.4 & 194.3 & 167.6 \\
August & 50.8 & 94.2 & 66 & 118.6 & 67.1 \\
September & 35.1 & 101.3 & 382.8 & 116.6 & 268 \\
October & 322.3 & 71.9 & 469.6 & 64 & 61.2 \\
November & 29.5 & 17.5 & 158.2 & 77.5 & 113.5 \\
December & 154.9 & 131.3 & 311.5 & 211.6 & 81 \\
\hline Annual total & 1340.3 & 1116.7 & 2309.5 & 1285.0 & 1610.1 \\
\hline † Data previously published [25]. & & &
\end{tabular}

\subsubsection{Statistics}

A two-way ANOVA was used on the mean M1 soil elements extracted with fixed variables being year $(\mathrm{Y})$, treatment and their associate interaction $\left(\mathrm{Y}^{*}\right.$ treatment). Results from topsoil M1 P and K concentrations obtained from 2016 to 2018 were published previously [25] and was merged with results collected in the period 2019-2020 to extend the field analysis and identify which treatment and extractable nutrients were significantly different. The soil results were limited to $\mathrm{M} 1$ extractable $\mathrm{P}, \mathrm{K}$, and Na because the remaining extractable nutrients (e.g., $\mathrm{Ca}, \mathrm{Mg}$, and $\mathrm{Cu}$ ) were similar to controls. A two-way ANOVA was also used to determine significance for corn grain and stover nutrient uptake with year and treatment as fixed variables. The reported results are limited to $\mathrm{P}, \mathrm{K}$ and Na uptake by corn stover. Corn grain nutrient composition showed no significant differences by year and treatment when compared with the control, so their results are not presented. All statistical analyses were determined using Sigma Stat v. 13 (SSPS Corp., Chicago, IL, USA) at a $p<0.05$ level of significance. Individual comparisons between fixed variables in the ANOVAs were done using the Holm-Sidek method.

\section{Results}

\subsection{Biochar Characteristics}

Acid digestion of biochars and SG compost revealed some interesting dissimilarities when plant macro- and micro-nutrient concentrations were compared (Table 1). As expected with wood-based feedstocks, $100 \%$ PC biochar had lower quantities of macro- 
(e.g., $\mathrm{P}, \mathrm{K}$, and $\mathrm{Ca}$ ) and micro-nutrients (e.g., $\mathrm{Cu}, \mathrm{Mn}$, and $\mathrm{Fe}$ ) compared to $100 \% \mathrm{PL}$ biochar. The $100 \%$ PC biochar and SG compost had relatively lower P, K and Na contents, making them ideal blending agents to reduce the relatively very high $\mathrm{P}$ concentrations $\left(33,000 \mathrm{mg} \mathrm{kg}^{-1}\right)$ in PL biochar (Table 1). Switchgrass compost had lower amounts of $\mathrm{Ca}$, and micronutrients (e.g., $\mathrm{Mn}, \mathrm{Cu}$, and $\mathrm{Fe}$ ) relative to $100 \% \mathrm{PC}$ biochar, but had relatively similar K and Mg concentrations to $100 \%$ PC biochar. The P content in SG compost (700 $\mathrm{mg} \mathrm{kg}^{-1}$ ) was approximately 2.5 -fold greater than that measured in $100 \%$ PC biochar (250 $\left.\mathrm{mg} \mathrm{kg}^{-1}\right)$.

\subsection{Soil Fertility}

Soil samples were collected from all plots before biochar application $\left(\mathrm{Y}_{1}-2016\right)$ and thereafter to determine annual soil nutrient dynamics along the experimental timeline $\left(Y_{2}\right.$ to $\left.Y_{5}-2020\right)$. The soil M1 P concentrations were presented on a year and treatment basis (Table 4). For soil M1 P concentrations, we found very significant treatment and year effect, including very significant interactions between these variables $(p<0.001$; Table 4$)$. The soil M1 K and Na concentrations were also presented on a year and treatment basis (Tables 4 and 5). For M1 K, there were significant $Y *$ treatment interaction (Table 5). The soil M1 Na concentrations also showed significant $Y *$ treatment interaction (Table 6).

Table 4. Topsoil (0-15 cm deep) Mehlich 1 extractable soil phosphorus (P) concentrations in $\mathrm{kg} \mathrm{ha}^{-1}$ by treatment and year (means from $n=4 ; \mathrm{Y}=$ year; $\mathrm{PC}=$ pine chip biochar; $\mathrm{PL}=$ poultry litter biochar; SG = switchgrass compost).

\begin{tabular}{cccccc}
\hline Treatment & $\mathbf{Y}_{\mathbf{1}}$ & $\mathbf{Y}_{\mathbf{2}}$ & $\mathbf{Y}_{\mathbf{3}}$ & $\mathbf{Y}_{\mathbf{4}}$ & $\mathbf{Y}_{\mathbf{5}}$ \\
\hline $\begin{array}{c}\text { Control (0 } \\
\text { biochar) }\end{array}$ & $86 \mathrm{a}, \mathrm{A}$ & $103 \mathrm{a}, \mathrm{A}$ & $101 \mathrm{a}, \mathrm{A}$ & $89 \mathrm{a}, \mathrm{A}$ & $59 \mathrm{~b}, \mathrm{~A}$ \\
100\% PC & $66 \mathrm{a}, \mathrm{A}$ & $93 \mathrm{~b}, \mathrm{~A}$ & $83 \mathrm{~b}, \mathrm{~A}$ & $72 \mathrm{a}, \mathrm{A}$ & $41 \mathrm{c}, \mathrm{A}$ \\
PC:PL 2:1 & $67 \mathrm{a}, \mathrm{A}$ & $265 \mathrm{~b}, \mathrm{~B}$ & $196 \mathrm{~b}, \mathrm{~B}$ & $220 \mathrm{~b}, \mathrm{~B}$ & $127 \mathrm{c}, \mathrm{B}$ \\
SG & $62 \mathrm{a}, \mathrm{A}$ & $78 \mathrm{a}, \mathrm{A}$ & $71 \mathrm{a}, \mathrm{A}$ & $63 \mathrm{a}, \mathrm{A}$ & $36 \mathrm{~b}, \mathrm{~A}$ \\
100\% PL & $73 \mathrm{a}, \mathrm{A}$ & $575 \mathrm{~b}, \mathrm{C}$ & $486 \mathrm{~b}, \mathrm{C}$ & $451 \mathrm{~b}, \mathrm{C}$ & $275 \mathrm{c}, \mathrm{C}$ \\
PC:PL 2:1 & $82 \mathrm{a}, \mathrm{A}$ & $100 \mathrm{a}, \mathrm{A}$ & $89 \mathrm{a}, \mathrm{A}$ & $72 \mathrm{a}, \mathrm{A}$ & $49 \mathrm{~b}, \mathrm{~A}$ \\
Source of & $p$ & & & & \\
variation & $<0.001$ & & & & \\
Treatment & $<0.001$ & & & & \\
Year (Y) & $<0.001$ & &
\end{tabular}

We observed that in $Y_{2}$ soil samples, application of 100\% PL and PC:PL 2:1 biochar resulted in relatively higher topsoil $(0-15 \mathrm{~cm}$ deep) $\mathrm{P}, \mathrm{K}$, and Na concentrations compared to the other treatments. For example, M1 P concentrations measured in the topsoil depth of the $100 \%$ PL treated plots were initially $73 \mathrm{~kg} \mathrm{ha}^{-1}$, but after treatment with PL biochar ranged between 265 and $575 \mathrm{~kg} \mathrm{ha}^{-1}$, Table 4). Blending the PL biochar with PC biochar in a 2:1 ratio reduced topsoil concentrations for these elements. Application of $100 \%$ PC biochar, PC biochar blended with SG (2:1), and the SG compost itself did not improve extractable M1 topsoil $\mathrm{P}, \mathrm{K}$ and Na concentrations compared to the control. In spite of receiving $\mathrm{P}$ and $\mathrm{K}$ fertilizer, topsoil $\mathrm{P}, \mathrm{K}$, and $\mathrm{Na}$ concentrations measured in all treatments declined with time. By $Y_{3}$ to $Y_{5}$, soil $\mathrm{K}$ and Na concentrations were of similar magnitude as compared to control topsoil concentrations. In contrast, plots treated with 100\% PL biochar and PC:PL 2:1 biochar blend had topsoil P concentrations that were still 2 to 4 -fold greater than the control. 
Table 5. Topsoil (0-15 cm deep) Mehlich 1 extractable soil potassium (K) concentrations in $\mathrm{kg} \mathrm{ha}^{-1}$ by treatment and year (means from $n=4 ; \mathrm{Y}=$ year; $\mathrm{PC}=$ pine chip biochar; $\mathrm{PL}=$ poultry litter biochar; SG = switchgrass compost).

\begin{tabular}{cccccc}
\hline Treatment & $\mathbf{Y}_{\mathbf{1}}$ & $\mathbf{Y}_{\mathbf{2}}$ & $\mathbf{Y}_{\mathbf{3}}$ & $\mathbf{Y}_{\mathbf{4}}$ & $\mathbf{Y}_{\mathbf{5}}$ \\
\hline $\begin{array}{c}\text { Control }(0 \\
\text { biochar) }\end{array}$ & $138 \mathrm{a}, \mathrm{A}$ & $136 \mathrm{a}, \mathrm{A}$ & $157 \mathrm{a}, \mathrm{A}$ & $195 \mathrm{a}, \mathrm{A}$ & $175 \mathrm{a}, \mathrm{A}$ \\
100\% PC & $155 \mathrm{a}, \mathrm{A}$ & $155 \mathrm{a}, \mathrm{A}$ & $156 \mathrm{a}, \mathrm{A}$ & $144 \mathrm{a}, \mathrm{A}$ & $160 \mathrm{a}, \mathrm{A}$ \\
PC:PL 2:1 & $132 \mathrm{a}, \mathrm{A}$ & $274 \mathrm{~b}, \mathrm{~B}$ & $207 \mathrm{c}, \mathrm{B}$ & $183 \mathrm{c}, \mathrm{A}$ & $174 \mathrm{c}, \mathrm{A}$ \\
SG & $156 \mathrm{a}, \mathrm{A}$ & $144 \mathrm{a}, \mathrm{A}$ & $139 \mathrm{a}, \mathrm{A}$ & $141 \mathrm{a}, \mathrm{A}$ & $160 \mathrm{a}, \mathrm{A}$ \\
100\% PL & $146 \mathrm{a}, \mathrm{A}$ & $493 \mathrm{~b}, \mathrm{C}$ & $303 \mathrm{c}, \mathrm{C}$ & $228 \mathrm{c}, \mathrm{B}$ & $206 \mathrm{c}, \mathrm{A}$ \\
PC:PL 2:1 & $136 \mathrm{a}, \mathrm{A}$ & $152 \mathrm{a}, \mathrm{A}$ & $153 \mathrm{a}, \mathrm{A}$ & $128 \mathrm{a}, \mathrm{A}$ & $157 \mathrm{a}, \mathrm{A}$ \\
Source of & $p$ & & & & \\
variation & $p$ & & & & \\
Treatment & $<0.001$ & & & & \\
Year $(\mathrm{Y})$ & $<0.001$ & & & & \\
$\mathrm{Y} *$ treatment & $<0.001$ & & & &
\end{tabular}

Lower-case letter indicates significant differences among mean values between years, while capital letter indicates significant differences among mean values between treatments using a two-way ANOVA at a $p<0.05$ level of significance.

Table 6. Topsoil (0-15 cm deep) Mehlich 1 extractable soil sodium (Na) concentrations in $\mathrm{kg} \mathrm{ha}^{-1}$ by treatment and year (means from $n=4 ; \mathrm{Y}=$ year; $\mathrm{PC}=$ pine chip biochar; $\mathrm{PL}$ = poultry litter biochar; SG = switchgrass compost).

\begin{tabular}{cccccc}
\hline Treatment & $\mathbf{Y}_{\mathbf{1}}$ & $\mathbf{Y}_{\mathbf{2}}$ & $\mathbf{Y}_{\mathbf{3}}$ & $\mathbf{Y}_{\mathbf{4}}$ & $\mathbf{Y}_{\mathbf{5}}$ \\
\hline Control $(0$ & $9.4 \mathrm{a}, \mathrm{A}$ & $11.7 \mathrm{a}, \mathrm{A}$ & $10.0 \mathrm{a}, \mathrm{A}$ & $7.2 \mathrm{a}, \mathrm{A}$ & $10.2 \mathrm{a}, \mathrm{A}$ \\
biochar) & $9.6 \mathrm{a}, \mathrm{A}$ & $14.1 \mathrm{a}, \mathrm{A}$ & $11.1 \mathrm{a}, \mathrm{A}$ & $6.2 \mathrm{a}, \mathrm{A}$ & $9.3 \mathrm{a}, \mathrm{A}$ \\
100\% PC & $9.9 \mathrm{a}, \mathrm{A}$ & $21.2 \mathrm{~b}, \mathrm{~A}$ & $13.4 \mathrm{a}, \mathrm{A}$ & $9.1 \mathrm{a}, \mathrm{A}$ & $14.7 \mathrm{a}, \mathrm{A}$ \\
PC:PL 2:1 & $9.9 \mathrm{a}, \mathrm{A}$ & $12.0 \mathrm{a}, \mathrm{A}$ & $10.1 \mathrm{a}, \mathrm{A}$ & $5.3 \mathrm{a}, \mathrm{A}$ & $12.5 \mathrm{a}, \mathrm{A}$ \\
SG & $12.7 \mathrm{a}, \mathrm{A}$ & $60.7 \mathrm{~b}, \mathrm{~B}$ & $26.9 \mathrm{c}, \mathrm{B}$ & $14.2 \mathrm{a}, \mathrm{A}$ & $16.3 \mathrm{a}, \mathrm{A}$ \\
100\% PL & $10.4 \mathrm{a}, \mathrm{A}$ & $14.6 \mathrm{a}, \mathrm{A}$ & $11.1 \mathrm{a}, \mathrm{A}$ & $6.1 \mathrm{a}, \mathrm{A}$ & $10.7 \mathrm{a}, \mathrm{A}$ \\
PC:PL 2:1 & $p$ & & & & \\
Source of & & & & & \\
variation & $<0.001$ & & & & \\
Treatment & $<0.001$ & & & & \\
Year $(\mathrm{Y})$ & $<0.001$ & & & & \\
$\mathrm{Y}$ * treatment & & & & & \\
\hline
\end{tabular}

Lower-case letter indicates significant differences among mean values between years, while capital letter indicates significant differences among mean values between treatments using a two-way ANOVA at a $p<0.05$ level of significance.

There was a significant $Y *$ treatment effect noted for $\mathrm{M} 1$ extractable $\mathrm{P}, \mathrm{K}$ and $\mathrm{Na}$ concentrations (Tables 4-6). Through $\mathrm{Y}_{2}$ to $\mathrm{Y}_{5}$, the M1 P concentrations were highest for soils treated with 100\% PL biochar and the PC:PL 2:1 blend compared to the other treatments. The significant $Y^{*}$ treatment interaction was also noted in topsoil M1 K concentrations especially in $Y_{2}$ and $Y_{3}$ (Table 5). There was likewise a significant $Y^{*}$ treatment interaction determined with M1 extractable Na concentrations $(p<0.001$; Table 6). This is evident by comparing $Y_{2}$ and $Y_{3}$ topsoil M1 Na concentrations after application of $100 \%$ PL biochar that ranged from 60.7 to $26.9 \mathrm{~kg} \mathrm{ha}^{-1}$ and in $Y_{4}$ to $Y_{5}$ ranged between 14.2 and $16.3 \mathrm{~kg} \mathrm{ha}^{-1}$, respectively (Table 6). Extractable $\mathrm{P}, \mathrm{K}$ and Na concentrations extracted from topsoils were of similar magnitude for the control and in the plots treated with $100 \%$ PC and PC:SG blend.

\subsection{Nutrient Uptake}

There was not a significant $Y^{*}$ treatment interaction ( $p=0.129$; Table 7$)$ that allows for a determination of which treatment and $Y$ had significant impacts on corn stover $P$ uptake results. Corn stover had significant increases in mean $P$ uptake concentration in $Y_{1}$ from 
all treatments except the $100 \%$ PC biochar and the control (Table 7). The P uptake influence was then limited in $Y_{2}$ to only soil treated with $100 \%$ PL biochar and with the PC:PL blend. In $Y_{3}$, the P uptake effect was significant only for $100 \%$ PL. By $Y_{4}$, there was no significant treatment effect (Table 7). Grouping the treatment means by years revealed that there was a significant decline in corn stover $\mathrm{P}$ uptake over time. This was consistent with the two-way ANOVA results showing a significant $Y *$ treatment interaction $(p<0.001)$.

Table 7. Annual corn stover phosphorus $(\mathrm{P})$ uptake $\left(\mathrm{kg} \mathrm{ha}^{-1}\right)$ after treatment with designer biochars (means computed from $n=4 ; \mathrm{Y}=$ year; $\mathrm{PC}=$ pine chip, $\mathrm{PL}=$ poultry litter; $\mathrm{SG}=$ switchgrass compost).

\begin{tabular}{cccccc}
\hline Treatment & $\mathbf{Y}_{\mathbf{1}}$ & $\mathbf{Y}_{\mathbf{2}}$ & $\mathbf{Y}_{\mathbf{3}}$ & $\mathbf{Y}_{\mathbf{4}}$ & Mean \\
\hline Control $(0$ & $8.25 \mathrm{a}, \mathrm{A}$ & $5.60 \mathrm{~b}, \mathrm{~A}$ & $3.48 \mathrm{~b}, \mathrm{~A}$ & $3.29 \mathrm{~b}, \mathrm{~A}$ & $5.16 \mathrm{~A}$ \\
biochar) & $6.83 \mathrm{a}, \mathrm{A}$ & $5.34 \mathrm{~b}, \mathrm{~A}$ & $4.25 \mathrm{~b}, \mathrm{~A}$ & $3.43 \mathrm{~b}, \mathrm{~A}$ & $4.96 \mathrm{~A}$ \\
100\% PC & $10.82 \mathrm{a}, \mathrm{B}$ & $9.05 \mathrm{a}, \mathrm{B}$ & $5.60 \mathrm{~b}, \mathrm{~A}$ & $4.26 \mathrm{~b}, \mathrm{~A}$ & $7.43 \mathrm{~B}$ \\
PC:PL 2:1 & $10.54 \mathrm{a}, \mathrm{B}$ & $4.99 \mathrm{~b}, \mathrm{~A}$ & $4.40 \mathrm{~b}, \mathrm{~A}$ & $2.95 \mathrm{~b}, \mathrm{~A}$ & $5.72 \mathrm{~A}$ \\
SG & $11.36 \mathrm{a} \mathrm{B}$ & $11.39 \mathrm{a}, \mathrm{B}$ & $8.17 \mathrm{~b}, \mathrm{~B}$ & $5.16 \mathrm{c}, \mathrm{A}$ & $9.02 \mathrm{~B}$ \\
100\% PL & $9.35 \mathrm{a}, \mathrm{B}$ & $5.75 \mathrm{~b}, \mathrm{~A}$ & $4.18 \mathrm{~b}, \mathrm{~A}$ & $3.21 \mathrm{~b}, \mathrm{~A}$ & $5.63 \mathrm{~A}$ \\
PC:SG 2:1 & $9.53 \mathrm{a}$ & $7.02 \mathrm{~b}$ & $5.01 \mathrm{c}$ & $3.72 \mathrm{~d}$ & \\
mean & $p$ & & & & \\
Source of & & & & & \\
variation & $<0.001$ & & & & \\
Year $(\mathrm{Y})$ & $<0.001$ & & & & \\
Treatment & & & & & \\
Y Treatment & 0.129 & & & & \\
\hline
\end{tabular}

Lower-case letter indicates significant differences among mean values between years, while capital letter indicates significant differences among mean values between treatments using a two-way ANOVA at a $p<0.05$ level of significance.

Stover $\mathrm{K}$ uptake was significantly influenced by $\mathrm{Y}$, treatment and a $\mathrm{Y} *$ treatment interaction $(p<0.001$; Table 8). The significant interaction effect between $Y$ and treatments makes it difficult to specifically discern which treatment had a significant impacted $\mathrm{K}$ uptake. All the same, we did measure the highest $\mathrm{K}$ uptake $\left(218.9 \mathrm{~kg} \mathrm{ha}^{-1}\right)$ by corn stover in $Y_{1}$ after treatment with 100\% PL biochar (Table 8). However, the corn stover K uptake concentrations declined from 218.9 in $Y_{1}$ to $122.5 \mathrm{~kg} \mathrm{ha}^{-1}$ in $Y_{3}$ in soil treated with $100 \%$ PL biochar. Potassium uptake by the other treatments ranged from 133.1 to $90.5 \mathrm{~kg}$ $\mathrm{ha}^{-1}$ in $\mathrm{Y}_{1}$ and also experienced concentration declines by $\mathrm{Y}_{4}$. (Table 8). The corn stover $\mathrm{K}$ concentration declines are corroborated by the significant $Y^{*}$ treatment interactions (Table 8).

Table 8. Annual corn stover potassium $(\mathrm{K})$ uptake $\left(\mathrm{kg} \mathrm{ha}^{-1}\right)$ after treatment with designer biochars (means from $n=4 ; \mathrm{Y}=$ year; $\mathrm{PC}=$ pine chip, $\mathrm{PL}=$ poultry litter; $\mathrm{SG}=$ switchgrass compost).

\begin{tabular}{ccccc}
\hline Treatment & $\mathbf{Y}_{\mathbf{1}}$ & $\mathbf{Y}_{\mathbf{2}}$ & $\mathrm{Y}_{\mathbf{3}}$ & $\mathbf{Y}_{\mathbf{4}}$ \\
\hline Control (0 & $90.5 \mathrm{a}, \mathrm{A}$ & $92.3 \mathrm{a}, \mathrm{A}$ & $81.9 \mathrm{a}, \mathrm{A}$ & $69.6 \mathrm{a}, \mathrm{A}$ \\
biochar) & $97.0 \mathrm{a}, \mathrm{A}$ & $85.4 \mathrm{a}, \mathrm{A}$ & $93.0 \mathrm{a}, \mathrm{A}$ & $74.9 \mathrm{a}, \mathrm{A}$ \\
100\% PC & $133.1 \mathrm{a}, \mathrm{B}$ & $131.5 \mathrm{a}, \mathrm{B}$ & $98.8 \mathrm{~b}, \mathrm{~A}$ & $80.7 \mathrm{~b}, \mathrm{~A}$ \\
PC:PL 2:1 & $131.1 \mathrm{a}, \mathrm{B}$ & $80.0 \mathrm{~b}, \mathrm{~A}$ & $92.3 \mathrm{~b}, \mathrm{~A}$ & $65.7 \mathrm{c}, \mathrm{A}$ \\
SG & $218.9 \mathrm{a}, \mathrm{C}$ & $142.1 \mathrm{a}, \mathrm{C}$ & $122.5 \mathrm{~b}, \mathrm{C}$ & $83.1 \mathrm{c}, \mathrm{A}$ \\
100\% PL & $92.1 \mathrm{a}, \mathrm{A}$ & $83.2 \mathrm{a}, \mathrm{A}$ & $87.0 \mathrm{a}, \mathrm{A}$ & $73.1 \mathrm{a}, \mathrm{A}$ \\
PC:SG 2:1 & $p$ & & & \\
Source of & $<0.001$ & & & \\
variation & $<0.001$ & & & \\
Year (Y) & $<0.001$ & & & \\
Treatment & & & \\
$\mathrm{Y}^{*}$ Treatment & & & & \\
\hline
\end{tabular}

$\overline{\text { Lower-case letter indicates significant differences among mean values between years, while capital letter indicates }}$ significant differences among mean values between treatments using a two-way ANOVA at a $p<0.05$ level of significance. 
Stover Na uptake concentrations was also significantly influenced by a $\mathrm{Y}^{*}$ treatment interaction $\left(p<0.001\right.$; Table 9). The significant interaction between $\mathrm{Y}^{*}$ treatment also muddles specific identification of which treatment had significant impact on mean $\mathrm{Na}$ uptake. However, we were able to determine that in $\mathrm{Y}_{1}$, corn stover Na uptake was highest after 100\% PL biochar and PC:PL 2:1 blend (1.915 and $0.808 \mathrm{~kg} \mathrm{ha}^{-1}$, respectively; Table 9) compared to the remaining treatments $\left(>0.340 \mathrm{~kg} \mathrm{ha}^{-1}\right)$. For all treatments in $\mathrm{Y}_{2}$ to $\mathrm{Y}_{4}, \mathrm{Na}$ uptake concentrations were of fairly similar in range $\left(0.111\right.$ to $\left.0.291 \mathrm{~kg} \mathrm{ha}^{-1}\right)$.

Table 9. Annual corn stover sodium $(\mathrm{Na})$ uptake $\left(\mathrm{kg} \mathrm{ha}^{-1}\right)$ after treatment with designer biochars (means computed from $n=4 ; \mathrm{Y}=$ year; $\mathrm{PC}=$ pine chip, $\mathrm{PL}=$ poultry litter; $\mathrm{SG}=$ switchgrass compost).

\begin{tabular}{ccccc}
\hline Treatment & $\mathbf{Y}_{\mathbf{1}}$ & $\mathbf{Y}_{\mathbf{2}}$ & $\mathbf{Y}_{\mathbf{3}}$ & $\mathbf{Y}_{\mathbf{4}}$ \\
\hline Control $(0$ & $0.308 \mathrm{a}, \mathrm{A}$ & $0.112 \mathrm{a}, \mathrm{A}$ & $0.214 \mathrm{a}, \mathrm{A}$ & $0.150 \mathrm{a}, \mathrm{A}$ \\
biochar) & $0.165 \mathrm{a}, \mathrm{A}$ & $0.194 \mathrm{a}, \mathrm{A}$ & $0.231 \mathrm{a}, \mathrm{A}$ & $0.110 \mathrm{a}, \mathrm{A}$ \\
100\% PC & $0.808 \mathrm{a}, \mathrm{B}$ & $0.199 \mathrm{~b}, \mathrm{~A}$ & $0.129 \mathrm{~b}, \mathrm{~A}$ & $0.158 \mathrm{~b}, \mathrm{~A}$ \\
PC:PL 2:1 & $0.340 \mathrm{a}, \mathrm{A}$ & $0.173 \mathrm{a}, \mathrm{A}$ & $0.111 \mathrm{a}, \mathrm{A}$ & $0.165 \mathrm{a}, \mathrm{A}$ \\
SG & $1.915 \mathrm{a}, \mathrm{C}$ & $0.214 \mathrm{~b}, \mathrm{~A}$ & $0.134 \mathrm{~b}, \mathrm{~A}$ & $0.291 \mathrm{~b}, \mathrm{~A}$ \\
100\% PL & $0.292 \mathrm{a}, \mathrm{A}$ & $0.180 \mathrm{a}, \mathrm{A}$ & $0.262 \mathrm{~b}, \mathrm{~A}$ & $0.118 \mathrm{a}, \mathrm{A}$ \\
PC:SG 2:1 & $p$ & & & \\
Source of & $<0.001$ & & & \\
variation & $<0.001$ & & & \\
Year (Y) & $<0.001$ & & & \\
Treatment & & & \\
$\mathrm{Y}^{*}$ Treatment & & & & \\
\hline
\end{tabular}

$\overline{\text { Lower-case letter indicates significant differences among mean values between years, while capital letter indicates }}$ significant differences among mean values between treatments using a two-way ANOVA at a $p<0.05$ level of significance.

\section{Discussion}

\subsection{Soil Fertility}

Applying the various designer biochars constructed to improve the inherently low fertility in the Goldsboro soil had different impacts on topsoil nutrient concentrations. As reported, PL biochar is enriched with plant macro- and micronutrients because these nutrients represent the element fraction not assimilated by animal digestion processes $[4,5]$. The unassimilated elements thus contribute to the PL nutrient pools in the animal wastebedding material. As such, pyrolysis of the 100\% PL feedstock into biochar results in nutrient-fortified biochar. Applying 100\% PL biochar to the Goldsboro topsoil resulted in fortifying M1 P, K and Na concentrations compared to the other amendments. In fact, application of $30 \mathrm{t} \mathrm{ha}^{-1}$ of $100 \%$ PL biochar resulted in mean topsoil M1 P concentrations ranging from 265 to $575 \mathrm{~kg} \mathrm{ha}^{-1}$, whereas prior to PL biochar application, background soil M1P concentrations in $\mathrm{Y}_{1}$ were $73 \mathrm{~kg} \mathrm{ha}^{-1}$ (Table 4). In comparison with soil test ratings vs. fertilizer recommendations [30], the M1 topsoil P concentrations measured in this study after $100 \%$ PL biochar application were several-fold higher when compared to the medium to high $\mathrm{P}$ concentration ranges $\left(46\right.$ to $112 \mathrm{~kg} \mathrm{ha}^{-1}$ ) recommended for corn production in sandy South Carolina Coastal Plain soils. The very high M1 P concentrations measured after $100 \%$ PL biochar application corroborated the need to reduce plant available P concentrations by constructing a blended amendment using the PC biochar. The 100\% PC biochar has a lower $\mathrm{P}$ concentration, so it was an ideal selection to dilute the high $\mathrm{P}$ content in the $100 \%$ PL biochar. Blending this designer biochar at a 2:1 PC:PL ratio caused reductions in topsoil mean M1 P concentrations ranging from 127 to $265 \mathrm{~kg} \mathrm{ha}^{-1}$ (in $\mathrm{Y}_{2}-\mathrm{Y}_{5}$; Table 4). The topsoil mean M1 P concentrations measured in this study are still greater than the high to very high M1 P soil test category ranking (e.g., 113 to $269 \mathrm{~kg} \mathrm{ha}^{-1}$ ) recommended for corn grain production in sandy SC coastal plain soils [30].

Similar trends occur with M1 topsoil K concentrations after treatment with 100\% PL biochar. Our measured topsoil M1 K contents when compared to recommended fertility ranges are in the high to very high soil test category range (205 to $265+\mathrm{kg} \mathrm{ha}^{-1}$ ) for corn 
grain production [30]. Likewise, our results in Table 5 showed that blending the $100 \%$ PL biochar with PC biochar reduced topsoil M1 K concentration to be comparable to the medium to very high category (174 to $265 \mathrm{~kg} \mathrm{ha}^{-1}$; [30]). The same trends occurred with M1 Na concentrations, in that, topsoil Na concentrations were highest after application of 100\% PL biochar, but were reduced by blending with PC biochar (Table 6). Another key trend noted is that topsoil M1 P, K, and Na concentrations in plots treated with 100\% PC biochar, PC:SG 2:1 biochar, or SG compost were of similar magnitude compared to the control (Tables 4-6). This was not surprising since biochar produced from hardwoods, softwoods, or grain crops have lower total $\mathrm{P}, \mathrm{K}$, and Na contents as compared to manurebased biochars [7].

As noted in Tables 4-6, there was a significant $Y *$ treatment influence on M1 topsoil $\mathrm{P}, \mathrm{K}$, and Na concentrations. The greatest soil M1 P, K, and Na nutrient concentrations were measured in $\mathrm{Y}_{2}$ after 100\% PL and the PC:PL 2:1 blended biochar application, but their concentrations gradually declined by $\mathrm{Y}_{3}$ to $\mathrm{Y}_{5}$ (Tables 3-5). As noted, application of the remaining biochar treatments did not result in a resounding increase in $\mathrm{P}, \mathrm{K}$, and $\mathrm{Na}$ concentrations in $Y_{2}$, as in fact, these three nutrient concentrations were similar to soil levels prior to biochar application $\left(\mathrm{Y}_{1}\right)$. Nevertheless, soil M1 P, K, and Na concentrations in all treatments declined as a function of experimental duration which explains the significant $\mathrm{Y}$ * treatment interaction (Tables 4-6).

We suspect that topsoil M1 P, K, and Na concentration declines with $\mathrm{Y}$ were related to a combination of crop uptake (as discussed in next section), to soil profile leaching from percolating rainfall, to disking in biochar, and by fertilizer placement. While soil $\mathrm{P}$ is known to be bound to Fe-, Al-oxyhydroxide phases and incorporated into soil organic matter [31], it usually does not readily leach in sandy soils.

Explanation for topsoil M1 K concentrations are a bit more complex. First, monovalent $\mathrm{K}$ ion is soluble in water and will leach through sandy soils [32,33]. Secondly, some biochars were more enriched with $\mathrm{K}$ than other biochars (Table 1). Thus, we speculate that the topsoil M1 K concentrations declines in plots treated with 100\% PL biochar and the PC:PL 2:1 biochar blend due to a combination of $\mathrm{K}$ leaching and with disking after application. All remaining treatments also experience M1 K concentration declines with time probably also explained by K leaching and corn stover uptake.

Sodium leaching through soils as a function of rainfall is a well-known process [31] and the decrease in Na concentrations with time are normal for this cation. However, a take home message is that the $100 \%$ PL litter biochar applied at $30 \mathrm{t} \mathrm{ha}^{-1}$ caused excess soil $\mathrm{P}, \mathrm{K}$ and $\mathrm{Na}$ concentrations in the initial corn crop cycle $\left(\mathrm{Y}_{1}\right)$, and their concentrations were reduced in subsequent corn crop cycles $\left(\mathrm{Y}_{3}-\mathrm{Y}_{5}\right)$. The remaining biochars and $\mathrm{SG}$ compost when added at $30 \mathrm{t} \mathrm{ha}^{-1}$ did not appreciably imbalance soil nutrient concentrations as compared to $100 \%$ PL biochar. Mixing the PC biochar with SG compost did not result in improvement in soil M1 P, K, and Na concentrations. In fact, their concentrations were similar to the controls. The finding of no improvement in soil nutrient availability after co-mixing biochar with a compost is similar to reports [34].

\subsection{Corn Grain and Stover Nutrient Uptake}

Our hypothesis to test in this investigation was to determine if additional nutrients supplied to soils through biochar and compost application would supplement existing nutrient pools and subsequently be taken up by corn grain and stover beyond the nutrients supplied by inorganic fertilizer and released during organic matter decomposition. Plant nutrient uptake was determined by digestion of corn grain and stover samples collected annually after four corn crop cycles. Among all the elements quantified in the digestates, no significant differences in nutrient uptake by corn grain were measured. With respect to corn stover compositional analyses, only $\mathrm{P}, \mathrm{K}$ and $\mathrm{Na}$ concentrations were found to be significant. Thus, we focused our explanation on corn stover uptake for these three nutrients.

With respect to corn stover $\mathrm{P}$ uptake in $\mathrm{Y}_{1}$, significant $\mathrm{P}$ uptake occurred after treatment with 100\% PL, PC:PL 2:1, PC:SG 2:1, and SG compost when compared to the control and 
$100 \%$ PC treatment (Table 7). In $\mathrm{Y}_{2}$, significant corn stover P uptake was limited to only 100\% PL and PC:PL 2:1 blend. By $Y_{3}$, the significant P uptake effect was limited to only corn stover grown in $100 \%$ PL biochar application, later on in $\mathrm{Y}_{4}$, there were no significant differences between treatments.

The impact of greater P uptake by corn stover was time and treatment dependent with a significant $p$ value of $<0.001$ determined for both variables (Table 7$)$. Therefore, if a soil plant available $P$ nutrient pool is in the low to moderate fertility range, then application of $100 \%$ PL biochar and PL biochar blended with PC biochar will supplement existing soil P concentrations. The supplemental $\mathrm{P}$ was then available for stover uptake. This was shown by comparing annual corn stover P uptake between treatments (Table 7). For example, corn stover P uptake in the Goldsboro soil control (no biochar applied) treatment ranged from 3.29 to $8.25 \mathrm{~kg} \mathrm{ha}^{-1}$ (Table 7). Corn grown in the control plots met P uptake requirements through inorganic $\mathrm{P}$ fertilizer and from soil $\mathrm{P}$ inorganic and organic nutrient sources. In comparison, applying biochar produced from 100\% PL and blended with PC biochar resulted in 2.57 to 3.11 (in $\mathrm{Y}_{1}$ ) and 0.97 to $1.87 \mathrm{~kg} \mathrm{ha}^{-1}$ (in $\mathrm{Y}_{4}$ ) more P uptake by corn stover compared to the control. The remaining treatments, when averaged over four corn growth cycles, did not show significantly more P uptake by stover compared to the control. In these four treatments, the lack of a significant differences with the control suggests that the corn crop relied on P supplied from inorganic fertilizer or from organic matter decomposition to a greater extent than that associated with the organic amendments.

With respect to K concentrations, application of 100\% PL biochar, the PC:PL blend and the switchgrass compost resulted in significant $\mathrm{K}$ uptake (Table 8). The significant uptake of $\mathrm{K}$ by corn stover is explained by the $100 \%$ PL biochar, its blend with PC biochar and the SG compost, all being enriched in total $\mathrm{K}$ (Table 1). The effect of additional $\mathrm{K}$ uptake is time dependent, since by $\mathrm{Y}_{2}$, corn stover $\mathrm{K}$ uptake from the SG treatment was similar to controls. In contrast to the results with SG treatment, significant $\mathrm{K}$ uptake occurred by corn stover in $\mathrm{Y}_{2}$ after application of $100 \% \mathrm{PL}$ and with the PC:PL blend. In $\mathrm{Y}_{3}$, more $\mathrm{K}$ was taken up by corn stover treated with only the $100 \%$ PL biochar. This indicates that K released from $100 \%$ PL biochar is still contributing to the soil K nutrient pool and benefiting corn nutritional demands. Potassium release into soil solution by weathering of PL biochar has been reported [10].

Potassium uptake by corn stover was also time dependent since in early years $\left(\mathrm{Y}_{1}\right.$ vs. $\left.\mathrm{Y}_{5}\right)$, there was sizable differences noted in the range of $\mathrm{K}$ uptake (92.1-218.9 vs. 65.7-83.1 $\mathrm{kg} \mathrm{ha}^{-1}$; Table 8) in plots treated with designer biochars. Simply comparing the size of the range differences in nutrient uptake between treatments offers some evidence of nutrient release dynamics by biochars. For example, there was a 2.5 -fold increase in stover $\mathrm{K}$ uptake in $\mathrm{Y}_{1}$ in plots treated with 100\% PL biochar vs. control (218.9 vs. $90.5 \mathrm{~kg} \mathrm{ha}^{-1}$; Table 8). By $\mathrm{Y}_{4}$, however, all treatments had near-similar mean $\mathrm{K}$ uptake ranges. This suggests that $\mathrm{K}$ released from all biochars had slowed to the point where plants were utilizing $\mathrm{K}$ from inorganic fertilizer, released from soil cation exchange sites, or released from organic matter decomposition. Here, examining the average $\mathrm{K}$ uptake in $\mathrm{Y}_{1}$ corn growth cycle, showed that $\mathrm{K}$ release from 100\% PL biochar and the PC:PL blend, respectively, resulted in 128.4 and $42.6 \mathrm{~kg}^{-1}$ more $\mathrm{K}$ uptake compared to the control. In $\mathrm{Y}_{4}$, the same comparison between these two treatments showed dramatic $\mathrm{K}$ uptake concentration declines to 11.1 and $13.5 \mathrm{~kg} \mathrm{ha}^{-1}$. These $\mathrm{K}$ uptake declines with time corroborate the noted $\mathrm{Y} *$ treatment interaction.

Under the sandy soil type, crop management, and climatic conditions in South Carolina, our results show sizable range differences especially after a one-time application of $100 \%$ PL biochar to the Goldsboro soil compared to the control. This finding suggests that applying $100 \%$ PL biochar to this soil will introduce a pulse of K available for corn stover uptake. However, the duration of this pulse impact on corn stover $\mathrm{K}$ uptake is short-termed. Because the impact of a single application of biochar has a short duration, repeated designer biochar application every three to four years would be ideal to supplement soil K concentrations. We realize that adding K-based fertilizer would be a less-expensive 
fertilizer option, however, there is the co-benefit of directly increasing other important plant nutrient (e.g., $\mathrm{Ca}, \mathrm{Mg}$, and $\mathrm{Zn}$ [7]) concentrations when biochar is applied.

There was also a significant $Y *$ treatment interaction noted with Na uptake by corn stover. The most significant differences in Na uptake was measured in corn stover grown in Goldsboro soil treated with 100\% PL biochar and the PC:PL 2:1 biochar blend. This trend was apparent by comparing the size differences between $\mathrm{Na}$ uptake between treatments by year reveal some interesting trends. For example, there was a 6-fold increase in stover Na uptake in $Y_{1}$ in plots treated with $100 \%$ PL biochar vs. control $\left(1.915 \mathrm{vs} .0 .308 \mathrm{~kg} \mathrm{ha}^{-1}\right.$; Table 9). By $Y_{4}$, however, all treatments had near-similar mean Na uptake ranges. This suggests that applying 100\% PL biochar results in a short-term spike in Na uptake that was limited to just $Y_{1}$ (Table 9). This finding confirms the significant $Y$ effect for Na uptake after biochar application $(p<0.001$; Table 9$)$.

We explain the significant $\mathrm{Y}$ effect for Na uptake by corn stover as related to the solubility of $\mathrm{Na}$ in soils. Here, the impact of $\mathrm{Na}$ uptake by stover from all biochars faded away with time probably due to Na leaching with percolating water from rainfall. Leaching of $\mathrm{Na}$ from soils in a normal process in the highly weathered Ultisols that formed in the humid climate of the Southeastern USA Coastal Plain region. In contrast, the increase in soil Na concentrations from $100 \%$ PL biochar application may be a concern in Ustic and Aridic moisture regimes in the Mid-Central and Southwestern USA agricultural region. In these regions, there is less rainfall to leach $\mathrm{Na}$, so $\mathrm{Na}$ accumulation from $100 \%$ PL biochar applications could become a sodic soil issue.

We highlight that more Na uptake by corn stover is important if the stover is harvested for biofuel processing within one year after 100\% PL biochar application. Sodium plus, alkali cations and other anions in stover will impact thermal conversion performance and costs during bio-oil production [35]. Residual alkali metals present in bio-oil can accelerate its aging and catalyze significant chemical changes during storage [36,37]. Moreover, alkali salts in bio-oils were found to deposit on the catalyst surface, causing poisoning of active sites and catalyst deactivation [37].

The PL-based designer biochars and the SG compost used in this study supplied additional $\mathrm{P}$ to the Goldsboro soil nutrient pools well beyond that supplied with inorganic fertilizer. Evidence was presented showing that additional P supplied by PL-based biochars were taken up by corn stover. Confounding interactions between treatment and year made determination of individual treatment effects for $\mathrm{K}$ and Na uptake difficult to interpret. However, simply comparing the range in $\mathrm{K}$ and $\mathrm{Na}$ corn stover uptake concentrations between $100 \%$ PL biochar and the control suggests enhanced uptake of these two nutrients.

No significant elemental uptake differences were found in corn grain. Measuring plant uptake of $\mathrm{P}, \mathrm{K}$, and $\mathrm{Na}$ over four crop cycles showed that the impact of these biochar supplied nutrients was time dependent. A few years after application, the benefit of additional $\mathrm{P}, \mathrm{K}$, and Na from PL-based biochars and SG compost fades causing plants to meet nutritional needs from existing soil nutrient pools.

If biochar is used to supplement soil fertilizer requirements for corn production in sandy South Carolina Coastal Plain soils, our results suggest that repeated applications may be needed. Others have suggested that repeated soil biochar applications should be furthered studied [38]. Repeated biochar applications to soil should be carefully considered, however, because this action may accelerate losses of indigenous soil organic matter by priming [39] and fertilizer $\mathrm{N}$ availability [40]. Potential losses of organic matter and $\mathrm{N}$ availability through repeated biochar applications could lower soil quality characteristics and impede achieving higher grain and biomass yields.

Author Contributions: Conceptualization, J.M.N. and G.C.S.; methodology, J.M.N. and D.W.W.; Formal analysis, J.M.N.; investigation, J.M.N., G.C.S., T.F.D., and D.W.W.; data curation, D.W.W.; writing-original draft preparation, J.M.N.; writing-review and editing, G.C.S. and D.W.W. All authors have read and agreed to the published version of the manuscript.

Funding: This research was funded by the U.S. Department of Agriculture (USDA). 
Institutional Review Board Statement: Not applicable.

Informed Consent Statement: Not applicable.

Acknowledgments: The authors thank Ken Stone for field fertilizer management, and Tillman Meyers and Hannah Rushmiller for their harvesting and soil sampling assistance. Mention of a trade names or commercial products in this article is solely for the purpose of providing specific information and does not imply recommendation or endorsement by the U.S. Department of Agriculture. The USDA is an equal opportunity employer.

Conflicts of Interest: The authors declare no conflict of interest.

\section{References}

1. Spokas, K.; Cantrell, K.B.; Novak, J.M.; Archer, D.W.; Ippolito, J.A.; Collins, H.P.; Boateng, A.A.; Lima, I.M.; Lamb, M.C.; McAloon, A.J.; et al. Biochar: A synthesis of its agronomic impact beyond carbon sequestration. J. Environ. Qual. 2012, 41, 973-989. [CrossRef]

2. Ding, Y.; Liu, Y.; Liu, S.; Li, Z.; Tan, X.; Huang, X.; Zeng, G.; Zhou, L.; Zheng, B. Biochar to improve soil fertility. Rev. Agron. Sustain. Develop. 2016, 36, 1-74. [CrossRef]

3. Blanco-Canqui, H. Biochar and soil physical properties. Soil Sci. Soc. Am. J. 2017, 84, 687-711. [CrossRef]

4. Novak, J.M.; Cantrell, K.B.; Watts, D.W. Compositional and thermal evaluation of lignocellulosic and poultry litter chars via high and low temperature pyrolysis. Bioenergy Res. 2013, 6, 114-130. [CrossRef]

5. Cantrell, K.B.; Hunt, P.G.; Uchimiya, M.; Novak, J.M.; Ro, K.S. Impact of pyrolysis temperature and manure source on physiochemical characteristics of biochar. Bioresour. Technol. 2012, 107, 484-497. [CrossRef]

6. Biederman, L.A.; Harpole, W.A. Biochar and its effects on plant productivity and nutrient cycling: A meta-analysis. GCB Bioenergy 2013, 5, 202-214. [CrossRef]

7. Ippolito, J.A.; Cui, L.; Kammann, C.; Wrage-Monnig, N.; Estavillo, J.M.; Fuertes-Mendizabal, T.; Sigua, G.; Novak, J.; Spokas, K.; Borchard, N. Feedstock choice, pyrolysis temperature and type influence biochar characteristics: A comprehensive meta-data analysis review. Biochar 2020, 2, 421-438. [CrossRef]

8. Hossain, M.; Bahar, M.; Sarkar, B.; Donne, S.; Ok, Y.S.; Palansooriya, K.; Kirkham, M.B.; Chowdhury, S.; Bolan, N. Biochar and its importance on nutrient dynamic in soil and plant. Biochar 2020, 2, 379-420. [CrossRef]

9. Laird, D.; Fleming, P.; Wang, B.; Horton, R. Biochar impact on nutrient leaching from a Midwestern Agricultural soil. Geoderma. 2010, 158, 436-442. [CrossRef]

10. Novak, J.M.; Sigua, G.; Watts, D.; Cantrell, K.; Shumaker, P.; Szogi, A.; Johnson, M.G.; Spokas, K.A. Biochars impact on water infiltration and water quality through a compacted subsoil layer. Chemosphere 2016, 142, 160-167. [CrossRef] [PubMed]

11. Gul, S.; Whalen, J.K. Biochemical cycling of nitrogen and phosphorus in biochar-amended soils. Soil Biol. Biochem. 2016, 103, 1-15. [CrossRef]

12. Luisba, S.; Odhiambo, J.; Ogola, J. Effect of biochar and phosphorus fertilizer application on soil fertility: Soil physical and chemical properties. Arch. Agron. Soil Sci. 2017, 63, 477-490. [CrossRef]

13. Jeffery, S.; Abalos, D.; Spokas, K.A.; Verheijen, G.A. Biochar effects on crop yields. In Biochar for Environmental Management, 2nd ed.; Lehmann, J., Joseph, S., Eds.; Earthscan Publishers: New York, NY, USA, 2015; pp. 282-326.

14. Laird, D.A.; Novak, J.M.; Collins, H.P.; Ippolito, J.A.; Karlen, D.L.; Lentz, R.; Sistani, K.; Spokas, K.; van Pelt, R. Multi-year and multi-location soil quality and crop biomass yield responses to hardwood fast pyrolysis biochar. Geoderma 2017, 289 , 46-53. [CrossRef]

15. Domingues, R.R.; Trugilho, P.F.; Silva, C.A.; de Melo, I.C.; Melo, L.C.; Margriotis, Z.M.; Sánchez-Monedero, M.A. Properties of biochar derived from wood and high nutrient biomasses with the aim of agronomic and environmental benefits. PLoS ONE 2017, 12, e0176884. [CrossRef] [PubMed]

16. Novak, J.M.; Cantrell, K.B.; Watts, D.W.; Busscher, W.J.; Johnson, M.G. Designing relevant biochars as soil amendments using lignocellulosic-based and manure-based feedstocks. J. Soils Sediments 2014, 14, 330-343. [CrossRef]

17. Busscher, W.J.; Novak, J.M.; Evans, D.E.; Watts, D.W.; Niandou, M.A.S.; Ahmedna, M. Influence of pecan biochar on physical properties of a Norfolk loamy sand. Soil Sci. 2010, 175, 10-16. [CrossRef]

18. Jeffery, S.; Abalos, D.; Prodana, M.; Bastos, A.C.; van Groenigen, J.W.; Hungate, B.A.; Verheijen, F. Biochar boosts tropical but not temperate crop yields. Environ. Res. Lett. 2017, 12, 053001. [CrossRef]

19. El-Naggar, A.; Lee, S.S.; Rinklebe, J.; Farooq, M.; Song, H.; Sarmah, A.K.; Zimmerman, A.R.; Ahmad, M.; Shaheen, S.M.; Ok, Y.K. Biochar application to low fertility soils: A review of current status, and future prospects. Geoderma 2019, 337, 536-554. [CrossRef]

20. Novak, J.M.; Lima, I.M.; Xing, B.; Gaskin, J.W.; Steiner, C.; Das, K.C.; Ahmedna, M.; Rehrah, D.; Watts, D.W.; Busscher, W.J.; et al. Characterization of designer biochar produced at different temperatures and their effects on a loamy sand. Ann. Env. Sci. 2009, 3, 195-206. Available online: https:/ / www.aes.northeastern.edu (accessed on 6 May 2021).

21. Novak, J.M.; Busscher, W.J. Selection and use of designer biochars to improve characteristics of southeastern USA coastal plain degraded soils. In Advanced Biofuels and Bioproducts; Springer Nature: New York, NY, USA, 2012; pp. 69-96. 
22. Hagemann, N.; Joseph, S.; Schmidt, H.P.; Kammann, C.I.; Harter, J.; Borch, T.; Young, R.B.; Varga, K.; Taherymoosavi, S.; Eliott, K.W.; et al. Organic coating on biochar explains its nutrient retention and stimulation of soil fertility. Nat. Commun. 2017, 8, 1089. [CrossRef]

23. Agegnehu, G.; Srivastava, A.K.; Bird, M.I. The role of biochar and biochar-compost in improving soil quality and crop performance: A review. Appl. Soil Ecol. 2017, 119, 156-170. [CrossRef]

24. Novak, J.M.; Sigua, G.C.; Ducey, T.F.; Watts, D.W.; Stone, K.C. Designer biochars impact on corn grain yields, biomass production, and fertility properties of a highly-weathered Ultisol. Environments 2019, 6, 64. [CrossRef]

25. Gee, G.W.; Bauder, J.W. Particle Size Analysis: Hydrometer Method. Chapter 15-Methods of Soil Analysis. Part 1-Physical and Mineralogical Methods; Book Series No. 5; American Society of Agronomy, Soil Science Society of America and Crop Science Society of America: Madison, WI, USA, 1984.

26. Daniels, R.B.; Buol, S.W.; Kleiss, H.J.; Ditzler, C.A. Soil Systems in North Carolina, Technical Bulletin 314; North Carolina State University: Raleigh, NC, USA, 1999.

27. ASTM. Petroleum Products, Lubricants, and Fossil Fuels: Gaseous Fuels, Coal, and Coke: American Society of Testing and Materials; (ASTM) International: Conshohocken, PA, USA, 2016.

28. Clemson University Agricultural Service Laboratory. Agricultural Methods and Analyses; Clemson University: Clemson, SC, USA, 2021; Available online: https:/ / www.clemson.edu/public/regulatory/ag-srvc-lab (accessed on 21 April 2014).

29. United States Department of Agriculture, National Resource Conservation Service, National Water and Climate Center, Historical Weather Data. 2021. Available online: https:/ / wcc.sc.egov.usda.gov/nwcc/site?sitenum=2037 (accessed on 25 April 2014).

30. Franklin, R.; Moore, K. Nutrient Management for SC Based Soil Test Results; Extension Circular 476; Clemson University: Clemson, SC, USA, 2002; Available online: https://www.clemson.edu/comm/manuals/publication/nutrient_management_for_south_ Carolina_ec476e.pdf (accessed on 6 May 2021).

31. Bohn, H.; McNeal, B.; O'Connor, G. Soil Chemistry; Wiley Inter-Science Publisher, John Wiley \& Sons: New York, NY, USA, 1979.

32. Kolahchi, Z.; Jalali, M. Effect of water quality on the leaching of potassium from sandy soil. J. Arid Environ. 2007, 68, 624-639. [CrossRef]

33. Wulff, F.; Schulz, A.; Jungk, A.; Claassen, N. Potassium fertilization on sandy soils in relation to soil test, crop yields, and K-leaching. Z. Pflanz. Bodenkd. 2011, 161, 591-599. [CrossRef]

34. Trupiano, D.; Cocozza, C.; Baronti, S.; Amendola, C.; Primo-Vaccari, F.; Lustrato, G.; Di Lonardo, S.; Fantasma, F.; Tognetti, R.; Scippa, G. The Effects of Biochar and Its Combination with Compost on Lettuce (Lactuca sativa L.) Growth, Soil Properties, and Soil Microbial Activity and Abundance. Int. J. Agron. 2017, 2017, 3158207. [CrossRef]

35. Mourant, D.; Wang, Z.; He, M.; Wang, X.S.; Garcia-Perez, M.; Ling, K. Mallee wood fast pyrolysis: Effects of alkali and alkaline earth metallic species on the yield and composition of bio-oil. Fuel 2011, 90, 2915-2922. [CrossRef]

36. Bridgwater, A.V. Review of fast pyrolysis of biomass and product upgrading. Biomass Bioenergy 2012, 38, 68-94. [CrossRef]

37. Ruddy, D.A.; Schaidle, J.A.; Ferrell, J.R.; Wang, J.; Moens, L.; Hensley, J.E. Recent advances in heterogeneous catalysts for bio-oil upgrading via "ex situ catalytic fast pyrolysis": Catalyst development through the study of model compounds. Green Chem. 2014, 16, 454-490. [CrossRef]

38. Nguyen, T.T.N.; Wallace, H.M.; Xu, C.Y.; Van Zweiten, L.; Weng, Z.H.; Xu, Z.; Che, R.; Tahmasbian, I.; Hu, H.W.; Bai, S.H. The effects of short term, long term and reapplication of biochar on bacteria. Sci. Total Environ. 2018, 636, 142-151. [CrossRef]

39. Luo, Y.; Lin, Q.M.; Durenkamp, M.; Kuzyakov, Y. Does repeated biochar incorporation induce further soil priming effect? J. Soil Sediments 2018, 18, 128-135. [CrossRef]

40. Huang, M.; Fan, L.; Chen, J.; Jiang, L.; Zou, Y. Continuous applications of biochar to rice: Effects on nitrogen uptake and utilization. Sci. Rep. 2018, 8, 11461. [CrossRef] [PubMed] 